\title{
Do children with congenital hypothyroidism exhibit abnormal cortical morphology?
}

\author{
Hayyah Clairman', Jovanka Skocic ${ }^{1}$, Julieta E. Lischinsky ${ }^{2}$ and Joanne Rovet ${ }^{1,3}$
}

\begin{abstract}
BACKGROUND: Given thyroid hormone (TH)'s essential role in multiple aspects of early brain development, children with congenital hypothyroidism $(\mathrm{CH})$ detected and treated early may still display subtle cognitive and behavioral impairments as well as brain abnormalities. However, effects on their cortical development are not yet known. We used an automated neuroimaging technique to determine if these children differ in cortical thickness (CT) from typically developing controls (TDC) and if the regions showing $C T$ differences reflect severity of initial hypothyroidism and predict later neuropsychological functioning.
\end{abstract}

METHODS: FreeSurfer Image Analysis Suite was used on archived MRI scans from $41 \mathrm{CH}$ and 42 TDC children aged 9-16 y. Vertex-based procedures were used to compare groups and perform correlations between CT and indices of disease severity and neuropsychological outcome.

RESULTS: The CH group showed multiple regions of cortical thinning or cortical thickening within right and left hemispheres relative to TDC. CT values were significantly correlated with early T4 and thyroid-stimulating hormone (TSH) levels and current neuropsychological test indices.

CONCLUSION: The developing cortex is sensitive to early TH loss in $\mathrm{CH}$. Different patterns of cortical thinning or cortical thickening among brain regions may reflect timing of TH deficiency relative to timing of cortical development.

C ongenital hypothyroidism $(\mathrm{CH})$ is a pediatric endocrine disorder that affects $\sim 1$ in 2,500 newborns (1) and is caused by a perinatal lack of thyroid hormone (TH). $\mathrm{CH}$ arises from abnormal development or function of the thyroid gland that produces $\mathrm{TH}$ or in a small proportion of cases, a defect in the hypothalamus or pituitary, which regulate the thyroid (2). Because TH is critical for many aspects of early brain development, $\mathrm{CH}$ was formerly a leading cause of mental retardation due to its long period before diagnosis and treatment (3). Since the advent of newborn screening, most affected yet asymptomatic children are now diagnosed and treated right after birth. However, they are still at risk for suboptimal development due to their brief period of TH deficiency. A considerable body of research on these children has shown that they have reduced IQ (3), poor school performance (4), behavior problems (5), and mild to moderate cognitive difficulties in language, visuospatial, sensorimotor, memory, and attention areas (6).

Rodent models of early hypothyroidism have been instrumental in pinpointing the specific manifestations of early $\mathrm{TH}$ deficiency within the developing brain. When TH is missing, fundamental neurobiological processes such as neurogenesis, neuronal migration, synaptogenesis, and myelination develop atypically (7), while structures such as hippocampus (8), striatum (9), and cortex $(10,11)$ are abnormal. Within the cortex, a late gestational/early postnatal TH insufficiency is associated with disturbed corticogenesis due to abnormal asymmetric division of neurons migrating toward the cortical surface (12). Since this aspect of corticogenesis gives rise to the particular cytoarchitectural feature known as cortical thickness (CT), it is possible that this may also be abnormal in children with $\mathrm{CH}$.

Advances in neuroimaging now make it possible to evaluate human cortical morphology. A commonly used tool is FreeSurfer Image Analysis Suite (Laboratory for Computational Neuroimaging, Martinos Center for Biomedical Imaging, Charlestown, MA), which via a set of automated algorithms, precisely reproduces gray/white and pial surfaces and measures CT by the distance between corresponding vertices on both surfaces (13). Research using this technique has shown distinct CT abnormalities in various pediatric conditions including autism (14), prematurity (15), attention deficit hyperactivity disorder (ADHD) (16), and fetal alcohol spectrum disorder (17).

Previously, we reported that children with $\mathrm{CH}$ show structural and/or functional abnormalities of the hippocampus $(18,19)$ but their cortex was not investigated. Therefore, we performed FreeSurfer (Laboratory for Computational Neuroimaging, Martinos Center for Biomedical Imaging) analyses on archived MRI scans of $\mathrm{CH}$ and typically developing control (TDC) groups. Our goals were to (i) identify brain regions where groups differed in CT, (ii) evaluate the relationships between patterns of cortical thinning or thickening and $\mathrm{CH}$ severity, and (iii) examine the relationships between $\mathrm{CT}$ and cognitive abilities in $\mathrm{CH}$. We hypothesized that the $\mathrm{CH}$ group will exhibit distinct $\mathrm{CT}$ abnormalities that will be

${ }^{1}$ Neurosciences and Mental Health Program, The Hospital for Sick Children, Toronto, Ontario, Canada; ${ }^{2}$ Institute for Biomedical Sciences, The George Washington University, Washington DC; ${ }^{3}$ Department of Pediatrics, University of Toronto, Toronto, Ontario, Canada. Correspondence: Joanne Rovet (joanne.rovet@sickkids.ca)

Received 13 August 2014; accepted 3 February 2015; advance online publication 17 June 2015. doi:10.1038/pr.2015.93 
associated both with severity of initial hypothyroidism and current weaknesses in neuropsychological functioning.

\section{RESULTS}

\section{Demographic, Neuropsychological, and Biomedical Results}

Table 1 presents demographic and neuropsychological test findings of $\mathrm{CH}$ and TDC groups. Groups did not differ in age, sex, handedness, socioeconomic status, or intracranial, gray matter, white matter, or cerebrospinal fluid volumes. $\mathrm{CH}$ scored significantly below TDC on the Wechsler Abbreviated Scale of Intelligence (WASI) Full Scale IQ, Vocabulary, and Matrix Reasoning (20) but not on the Rey-Osterrieth Complex Figure Test (REY-O) (21).

Table 2 contains biomedical data for the $\mathrm{CH}$ group. Findings revealed a mean $\pm \mathrm{SD}$ thyroxine value at diagnosis (T4dx) of $58.9 \pm 39.4 \mathrm{nmol} / \mathrm{l}$, which is in the hypothyroid range (normal range $=65.0-165 \mathrm{nmol} / \mathrm{l})$. Their thyroid stimulating hormone levels at diagnosis (TSHdx) were well above normal (median $=265.1 \mathrm{mU} / \mathrm{l}$; normal range $=0.5-5.0 \mathrm{mU} / \mathrm{l})$ and

Table 1. Mean $( \pm S D)$ demographic and neuropsychological test data for $\mathrm{CH}$ and control groups

\begin{tabular}{|c|c|c|c|}
\hline & $\begin{array}{l}\text { CH group } \\
(n=42)\end{array}$ & $\begin{array}{l}\text { Control group } \\
\qquad(n=42)\end{array}$ & $P$ value \\
\hline Age (years) & $12.4 \pm 1.8$ & $12.0 \pm 1.6$ & ns \\
\hline Sex (males) & 20 & 20 & ns \\
\hline $\begin{array}{l}\text { Socioeconomic } \\
\text { status level }^{a}\end{array}$ & $1.9 \pm 0.92$ & $2.0 \pm 0.94$ & ns \\
\hline Handedness $(\% \mathrm{RH})^{\mathrm{b}}$ & 83 & 88 & ns \\
\hline Intracranial volume & $1,709.6 \pm 179.0^{c}$ & $1,735.8 \pm 188.0$ & ns \\
\hline Gray matter volume & $847.8 \pm 96.0^{c}$ & $869.4 \pm 84.5$ & ns \\
\hline White matter volume & $428.0 \pm 56.8^{c}$ & $420.3 \pm 45.4$ & ns \\
\hline $\begin{array}{l}\text { Cerebrospinal fluid } \\
\text { volume }\end{array}$ & $433.8 \pm 98.7^{c}$ & $446.1 \pm 131.5$ & ns \\
\hline IQ & $105.5 \pm 10.1$ & $115.8 \pm 9.8$ & 0.000 \\
\hline WASI vocabulary & $10.8 \pm 2.3$ & $13.2 \pm 2.5$ & 0.000 \\
\hline WASI matrix reasoning & $11.1 \pm 1.8$ & $12.2 \pm 1.6$ & 0.004 \\
\hline REY-O copy ${ }^{d}$ & $-0.421 \pm 1.1$ & $-0.178 \pm 1.0$ & ns \\
\hline REY-O delayed ${ }^{d}$ & $-0.980 \pm 1.2$ & $-0.538 \pm 1.0$ & ns \\
\hline
\end{tabular}

REY-O, Rey-Osterrieth Complex Figure Test; WASI, Wechsler Abbreviated Scale of Intelligence.

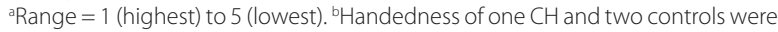
unknown. ${ }^{c} n=41$ as one $\mathrm{CH}$ scan was excluded from analyses due to excessive motion. ${ }^{\mathrm{d}} \mathrm{CH}$ group, $n=41$; control group, $n=38$.

Table 2. Congenital hypothyroidism group's biomedical data at time of diagnosis

\begin{tabular}{|c|c|c|c|}
\hline & Value & Range & $\begin{array}{l}\text { Normal } \\
\text { range }\end{array}$ \\
\hline $\mathrm{T} 4 \mathrm{dx}(\mathrm{nmol} / \mathrm{l})^{\mathrm{a}}$ & $58.9 \pm 39.4$ & $8-161$ & $65-165$ \\
\hline $\mathrm{TSHdx}(\mathrm{mU} / \mathrm{l})^{\mathrm{b}}$ & 265.1 & $14.47-1181.9\left(107-536^{c}\right)$ & $0.5-5.0$ \\
\hline $\begin{array}{l}\text { Age (days) at start } \\
\text { of treatment }^{\mathrm{a}}\end{array}$ & $16.8 \pm 13.3$ & $7-63$ & - \\
\hline \multicolumn{2}{|c|}{ Starting dose $(\mu \mathrm{g} / \mathrm{kg})^{\mathrm{a}} 10.38 \pm 1.35$} & $8.33-13.5$ & - \\
\hline
\end{tabular}

aData presented as mean \pm SD. ' ${ }^{\circ}$ Data presented as median. Intraquartile range. quite variable (range $=14.5-1,182 \mathrm{mU} / \mathrm{l}$ ), with two cases having TSHdx values above $1,000 \mathrm{mU} / \mathrm{l}$. Most children began treatment within 2 wk of birth (median $=13 \mathrm{~d}$; range $=7-63 \mathrm{~d}$ ) and their mean \pm SD starting dose of L-T4 was $10.38 \pm 1.35 \mu \mathrm{g} /$ $\mathrm{kg}$, with $71 \%$ of dosages above $10 \mu \mathrm{g} / \mathrm{kg} /$ day.

\section{Cortical Morphometry Results}

Table 3 lists the regions that differentiated $\mathrm{CH}$ and TDC groups while Figure 1 provides their CT-difference maps. As per heat bars, regions showing significant thinning in $\mathrm{CH}$ (i.e., $\mathrm{CH}<\mathrm{TDC}$ ) are depicted in blue and significant thickening, in red $(\mathrm{CH}>\mathrm{TDC})$. These results are based on $41 \mathrm{CH}$ cases and 42 TDC since one CH child's scan was excluded due to excessive motion.

Relative to TDC, $\mathrm{CH}$ demonstrated significant thinning in four left- and seven right-hemisphere regions. These were located in: (i) frontal and temporal poles and superior frontal gyri bilaterally, (ii) superior parietal gyrus of the left hemisphere, and (iii) middle frontal gyrus and sulcus, inferior temporal gyrus, and precuneus of the right hemisphere. In contrast, $\mathrm{CH}$ demonstrated significant thickening relative to TDC in the left central sulcus, supramarginal sulcus, calcarine sulcus, and occipital pole and the right medial orbitofrontal and middle and superior occipital sulci. Most regions showing thinning in $\mathrm{CH}$ were gyri, whereas all regions showing thickening were sulci.

\section{Effects of Early Disease Indices}

Table 4 presents the significant correlations between CT and T4dx while Figure 2 presents brain maps showing significant correlations with T4dx. Figure 3 also shows sample positive and negative correlations for left lateral fissure and right posctentral gyrus respectively. Note that in brain-map figures of correlations, colors are based on heat bars and represent strength and direction of the correlation, not degree of thinning/thickening (as in Figure 1). Since a low T4dx value signifies severe hypothyroidism and a higher value, normalto-near normal TH levels, regions shown in red (positive correlation) indicate the cortex is thinner than normal when hypothyroidism was more severe and regions in blue (negative correlation), a thicker than normal cortex. The following regions were thinner among the $\mathrm{CH}$ children with lower T4dx values (positive correlation): superior frontal gyrus bilaterally, cingulate gyrus, lateral fissure posterior segment, and superior temporal gyrus of the left hemisphere, and rectus gyrus and inferior parietal gyrus of the right hemisphere. In contrast, the following regions were thicker when $\mathrm{T} 4 \mathrm{dx}$ values were low (negative correlation): left middle frontal sulcus and calcarine sulcus and right postcentral gyrus and inferior temporal sulcus.

Table 5 shows opposite effects for TSHdx, which when high signifies severe hypothyroidism. Thus in Figure 4, red indicates regions of increased thickening with more severe hypothyroidism and blue, regions of increased thinning. Figure 5 additionally provides sample correlations for a region showing thickening and one showing thinning. Only 


\section{Articles $\mid$ Clairman et al.}

Table 3. FreeSurfer findings showing regions significantly differentiating $\mathrm{CH}$ and control groups in cortical thickness ${ }^{\mathrm{a}}$

\begin{tabular}{|c|c|c|c|c|c|c|}
\hline Hemisphere & Lobe/region & Structure & $\begin{array}{c}\text { Talairach } \\
\text { coordinates }(x, y, z)\end{array}$ & \# vertices & Size $\left(\mathrm{mm}^{2}\right)$ & $P$ value \\
\hline \multirow[t]{3}{*}{ Left } & Frontal & Rostral middle frontal sulcus (frontal pole) & $-21,56,-1$ & 142 & 108.6 & 0.0002 \\
\hline & Parietal & Superior parietal gyrus & $-12,-57,57$ & 170 & 73.8 & 0.0044 \\
\hline & Temporal & Temporal pole & $-32,-3,-31.5$ & 118 & 60.1 & 0.0204 \\
\hline \multirow{4}{*}{ Right } & Frontal & Superior frontal gyrus & $9,25,52$ & 290 & 135.7 & 0.0002 \\
\hline & Frontal & Frontal pole & $18,57,10$ & 144 & 119.4 & 0.0002 \\
\hline & Parietal & Precuneus & $6,-59,53$ & 186 & 75.7 & 0.0034 \\
\hline & Temporal & Temporal pole & $34,-3,-36$ & 173 & 99.3 & 0.0004 \\
\hline \multirow{2}{*}{ Left } & Occipital & Pericalcarine sulcus (occipital pole) & $-15,-92,-1$ & 90 & 75.1 & 0.0040 \\
\hline & Parietal & Inferior parietal (supramarginal sulcus) & $-44,-23,22$ & 138 & 57.6 & 0.0264 \\
\hline \multirow[t]{3}{*}{ Right } & Frontal & Medial orbitofrontal sulcus & $14,27,-19$ & 110 & 58.8 & 0.0334 \\
\hline & Occipital & Middle occipital sulcus & $28,-83,7$ & 171 & 114.3 & 0.0002 \\
\hline & Occipital & Superior occipital sulcus & $27,-64,27$ & 308 & 144.4 & 0.0002 \\
\hline
\end{tabular}

$\mathrm{CH}$, congenital hypothyroidism.

a Significance is at the $P \leq 0.05$ level.
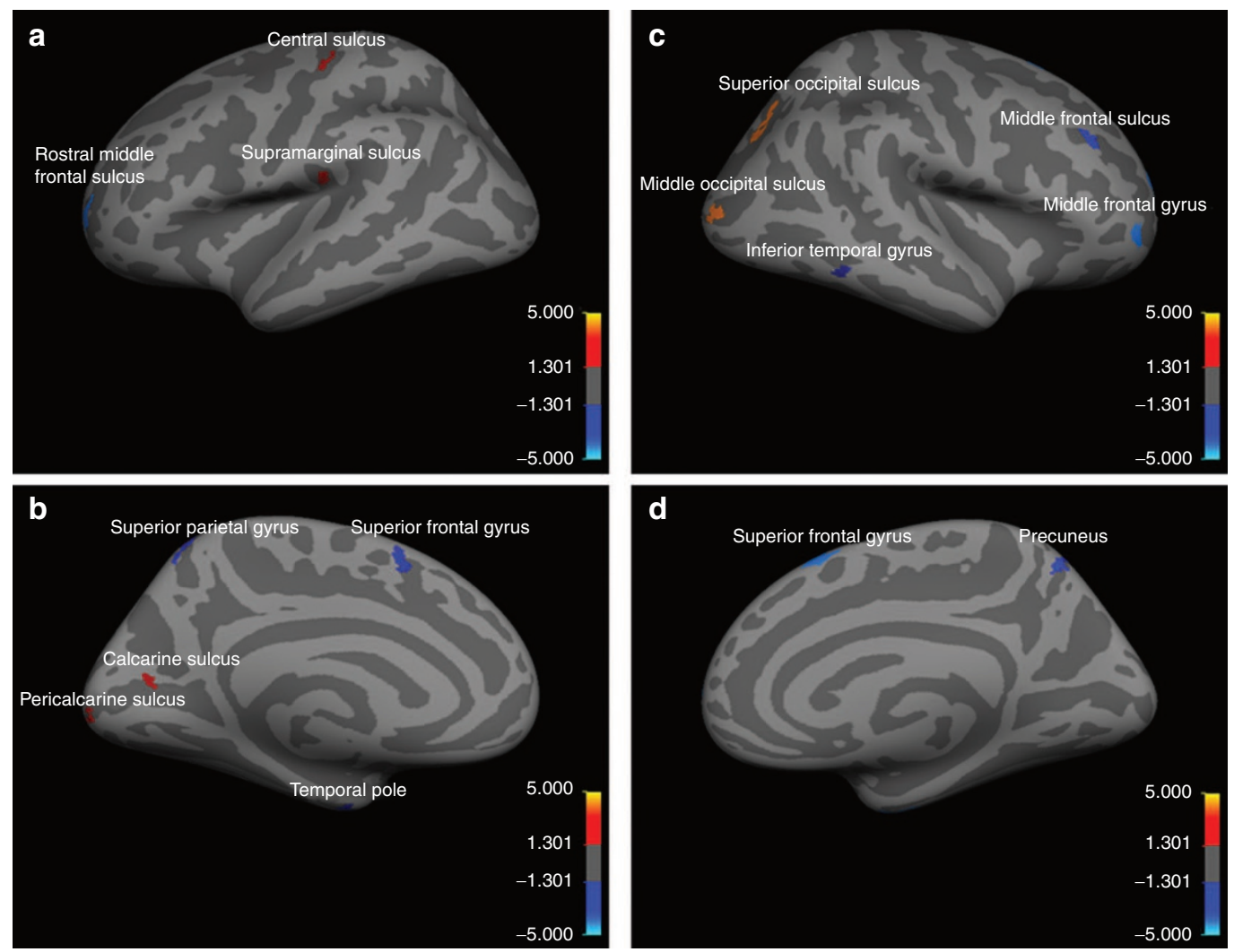

Figure 1. FreeSurfer (Laboratory for Computational Neuroimaging, Martinos Center for Biomedical Imaging, Charlestown, MA) difference map for cortical thickness. Red tones denote regions where congenital hypothyroidism $(\mathrm{CH})$ participants are thicker relative to typically developing controls (TDC). Blue shades denote regions where $\mathrm{CH}$ participants are thinner relative to TDC. Left hemisphere lateral (a) and medial (b) views; right hemisphere lateral (c) and medial (d) views. A $P$ value of $\leq 0.05$ was used for statistical significance. 
Table 4. Regions showing significant correlations between $T 4$ at diagnosis and cortical thickness ${ }^{\mathrm{a}}$

\begin{tabular}{|c|c|c|c|c|c|c|}
\hline Hemisphere & Lobe/region & Structure & $\begin{array}{c}\text { Talairach } \\
\text { coordinates }(x, y, z)\end{array}$ & \# vertices & Size $\left(\mathrm{mm}^{2}\right)$ & $P$ value \\
\hline \multicolumn{7}{|c|}{ T4 positively correlated with CT } \\
\hline \multirow{2}{*}{ Left } & Frontal/Parietal & Cingulate gyrus & $-5,4,30$ & 158 & 56.9 & 0.0216 \\
\hline & Occipital/Parietal & Lateral fissure (posterior segment) & $-41,-37,20.5$ & 236 & 89.3 & 0.0006 \\
\hline \multirow{2}{*}{ Right } & Frontal & Superior frontal gyrus & $8,-3,55$ & 124 & 65.3 & 0.0074 \\
\hline & Parietal & Inferior parietal gyrus (angular) & $41,-60,43$ & 143 & 70.5 & 0.0040 \\
\hline \multicolumn{7}{|c|}{ T4 negatively correlated with CT } \\
\hline Left & Frontal & Middle frontal sulcus & $-27,45,4$ & 153 & 89.2 & 0.0006 \\
\hline
\end{tabular}

$\mathrm{CH}$, congenital hypothyroidism; CT, cortical thickness.

apositive correlation indicates lower T4 (more severe $\mathrm{CH}$ ) at diagnosis leads to thinning of cortical regions listed, negative correlation indicates lower T4 at diagnosis leads to thickening $P$ value for significance is $<0.05$.

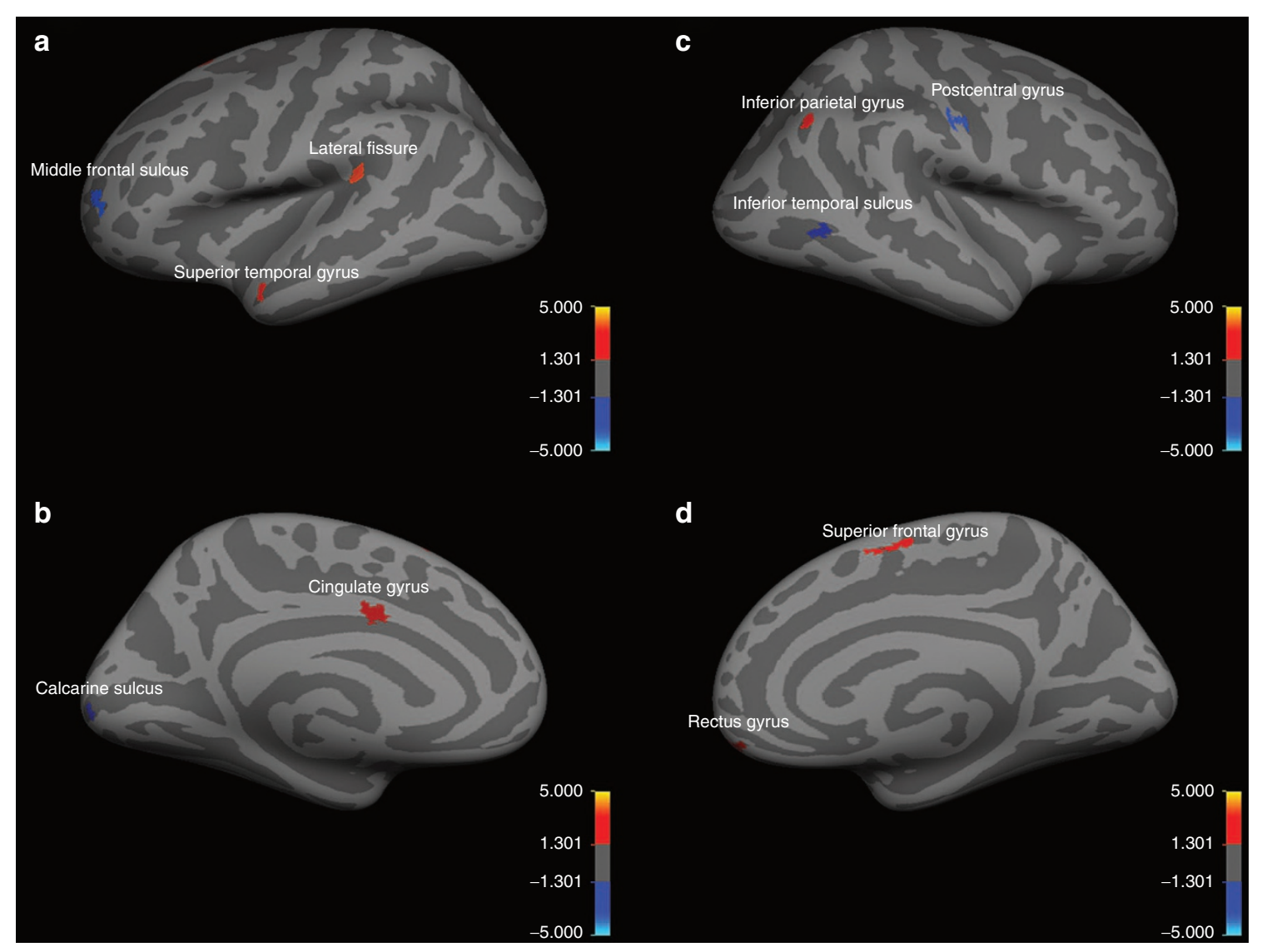

Figure 2. Correlation with T4 at diagnosis. Left hemisphere lateral (a) and medial (b) views; right hemisphere lateral (c) and medial (d) views; red tones indicate regions of thinning with low T4dx; blue shades show regions of thickening when child was more hypothyroid (i.e., T4dx was lower).

two regions were found to be thinner when TSHdx values were higher signifying more severe hypothyroidism: right middle frontal sulcus and right parieto-occipital sulcus (Figure 5). In contrast, 16 regions were found to be thicker in $\mathrm{CH}$ than controls (Table 5): left paracentral gyrus (3 regions), precentral gyrus, superior frontal gyrus (Figure 5), cingulate sulcus, calcarine sulcus, intraparietal sulcus, and temporal pole and right superior frontal gyrus (3 regions), 


\section{Articles | Clairman et al.}

a

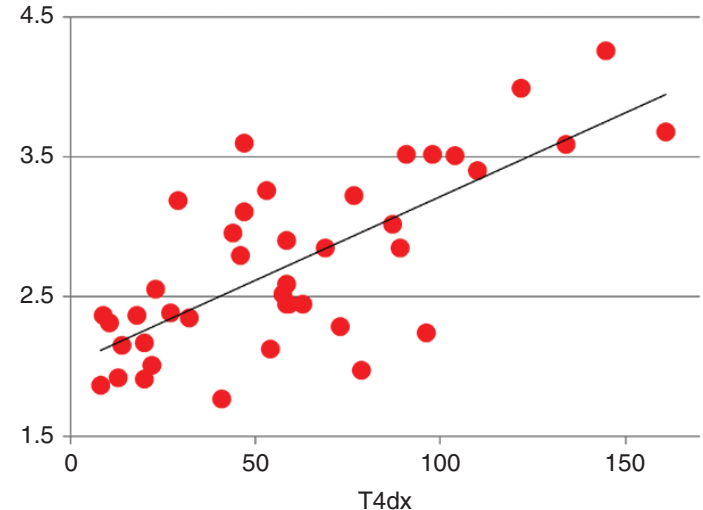

b

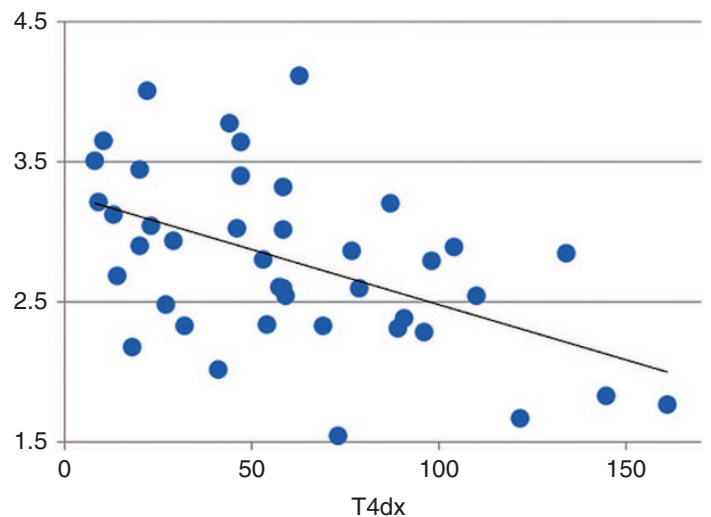

Figure 3. Sample positive (a) and negative (b) correlations showing regions that were thinner (red) or thicker (blue) when T4dx values were lower. Figure on left is of left lateral fissure posterior segment ( $-41-3720.5), 236$ vertices, $P=0.0006$; figure on right is of right postcentral gyrus (56.5 -1337 ), 211 vertices, $P=0.0008$. Horizontal axis shows children's T4 values at diagnosis (lower $=$ worse); vertical axis shows cortical thickness in $\mathrm{mm}$.

Table 5. Regions showing significant correlations between TSH at diagnosis and cortical thickness ${ }^{\mathrm{a}}$

\begin{tabular}{|c|c|c|c|c|c|c|}
\hline Hemisphere & Lobe/region & Structure & $\begin{array}{c}\text { Talairach } \\
\text { coordinates }(x, y, z)\end{array}$ & \# vertices & Size $\left(\mathrm{mm}^{2}\right)$ & $P$ value \\
\hline \multicolumn{7}{|c|}{ TSH negatively correlated with CT } \\
\hline Right & Occipital/Parietal & Parieto-occipital sulcus & $21,-66,29$ & 110 & 69.5 & 0.0044 \\
\hline \multicolumn{7}{|c|}{ TSH positively correlated with $C T$} \\
\hline \multirow{4}{*}{ Left } & Central & Paracentral gyrus & $-18,-38,67$ & 139 & 60.6 & 0.0128 \\
\hline & Frontal & Cingulate sulcus & $-12,16.5,30$ & 287 & 117.6 & 0.0002 \\
\hline & Frontal & Precentral gyrus & $-31.5,-15,63$ & 173 & 73.7 & 0.0024 \\
\hline & Frontal & Superior frontal gyrus & $-7,20.5,52$ & 149 & 79.1 & 0.0012 \\
\hline \multirow[t]{7}{*}{ Right } & Frontal & Superior frontal gyrus & $10,62,9$ & 150 & 113.1 & 0.0002 \\
\hline & Frontal & Superior frontal gyrus & $11,52,28$ & 158 & 95.7 & 0.0002 \\
\hline & Frontal & Superior frontal gyrus & $12,41,39$ & 104 & 59.4 & 0.0156 \\
\hline & Frontal/Parietal & Cingulate sulcus & $11,6,35$ & 221 & 85.8 & 0.0006 \\
\hline & Occipital & Middle occipital gyrus & $38,-76,18$ & 94 & 62.5 & 0.0106 \\
\hline & Parietal & Postcentral gyrus & $56,-12,39$ & 236 & 56.2 & 0.0006 \\
\hline & Temporal & Superior temporal sulcus & $51,-39,-2$ & 139 & 63.6 & 0.0088 \\
\hline
\end{tabular}

$\mathrm{CH}$, congenital hypothyroidism; $\mathrm{CT}$, cortical thickness; $\mathrm{TSH}$, thyroid-stimulating hormone.

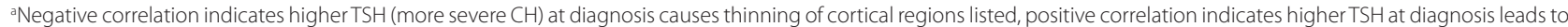
thickening; $P$ value for significance is $<0.05$.

cingulate sulcus, middle occipital gyrus, postcentral gyrus, and superior temporal sulcus.

In addition, a subgroup analysis compared athyrosis $(n=13)$ and ectopic $(n=20)$ etiologic subgroups; the dyshormonogenesis subgroup was not included due to its small number of cases. Results revealed that the right occipital pole was significantly thinner in the athyrotic group $(P=0.0002)$. Conversely, these children showed thickening in diverse regions of both hemispheres relative to those with ectopic glands (see Figure 6): left middle frontal gyrus $(P=0.049)$ and collateral transverse sulcus $(P=0.026)$ and right occipito-temporal gyrus (two regions, $P=0.009$ and $P=0.003)$, superior occipital gyrus $(P=0.0002)$, intraparietal sulcus $(P=0.0066)$, and inferior parietal gyrus $(P=0.015)$. 


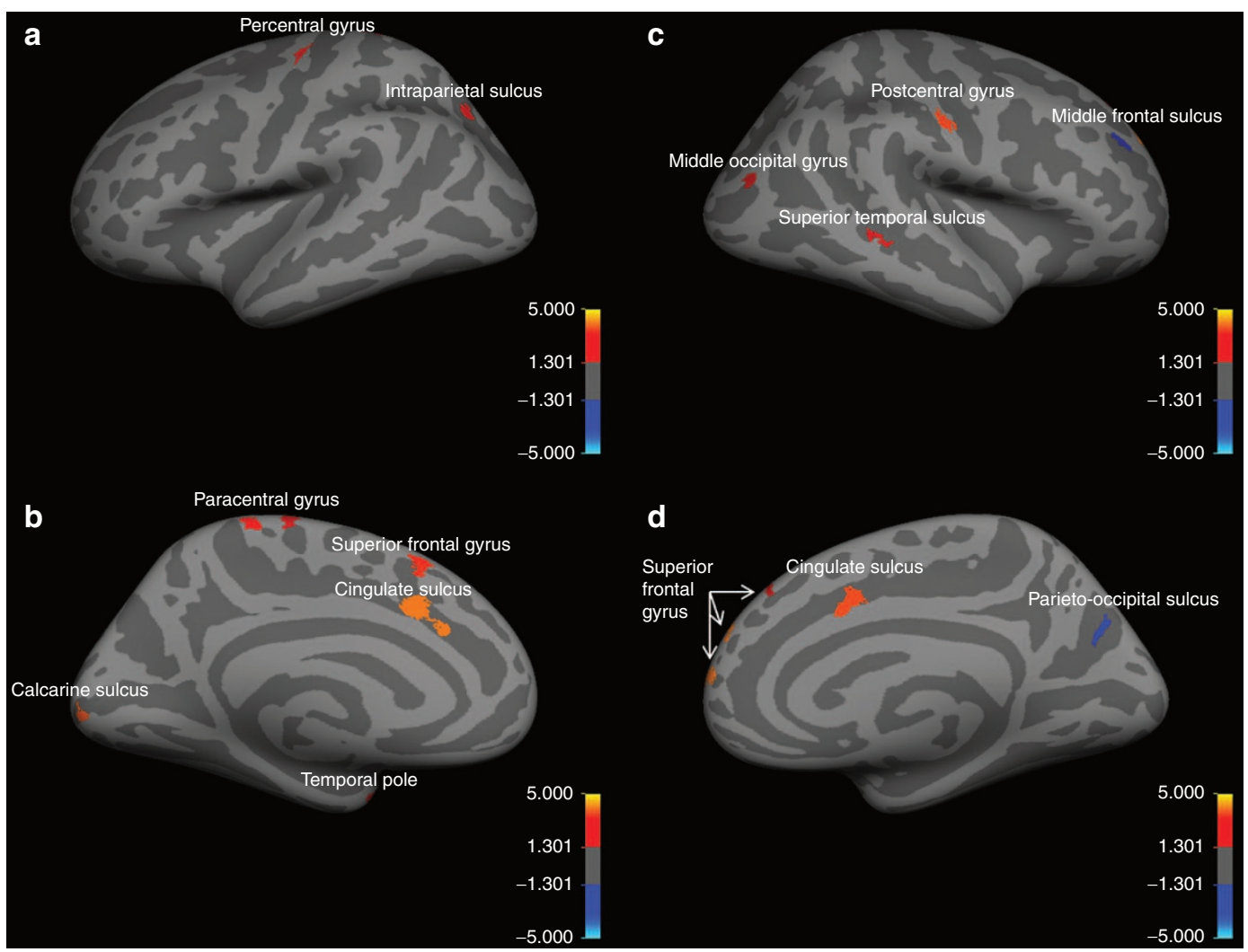

Figure 4. Correlation with thyroid-stimulating hormone (TSH) at diagnosis. Left hemisphere lateral (a) and medial (b) views; right hemisphere lateral (c) and medial (d) views; blue shades indicate regions of thinning with high TSH; red tones indicate regions of thickening when TSH was high.

a

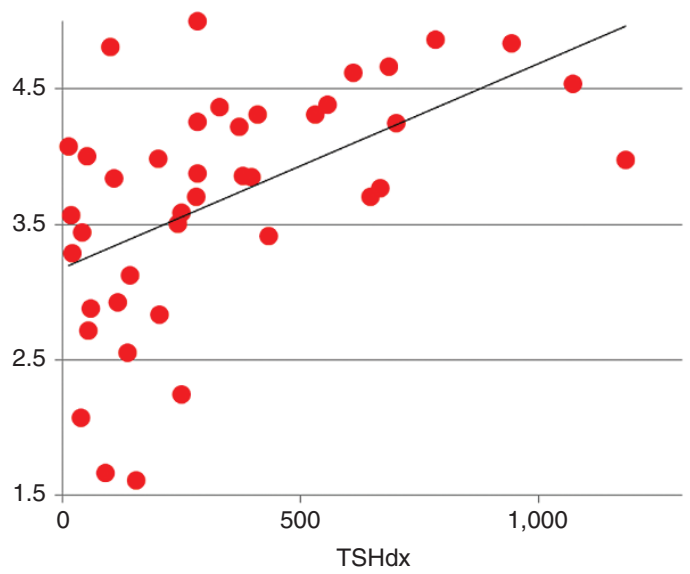

b

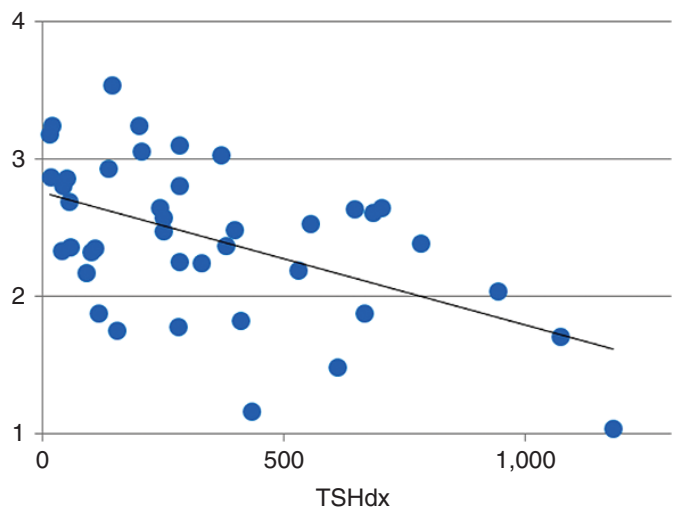

Figure 5. Sample positive (a) and negative (b) correlations showing regions that were thicker (red) or thinner (blue) when child was more hypothyroid (i.e., TSHdx was higher). Figure on left is of right superior frontal gyrus (10 62 9), 150 vertices, $P=0.0002$; figure on right is of right parieto-occipital sulcus (21-66 29), 110 vertices, $P=0.004$. Horizontal axis shows children's TSH values at diagnosis (higher $=$ worse); vertical axis shows cortical thickness in millimeters. TSH, thyroid-stimulating hormone.

\section{Correlations With Neuropsychological Test Results}

Table 6 lists, for $\mathrm{CH}$ children, the significant correlations between CT and neuropsychological test results on tasks differentiating groups (see Table 1). All correlations were negative signifying worse performance when cortices were thicker. For full-scale IQ, lower scores were associated with thickening of the left superior frontal gyrus and rectus gyrus and right orbital gyrus, and at a trend level $(P<0.01)$, left middle frontal, anterior cingulate gyrus, collateral sulcus, and another but different region of the right orbital gyrus. Lower WASI Vocabulary scores were associated with thickening of the left middle and superior frontal gyri, left collateral transverse sulcus, and right orbital gyrus. Lower WASI Matrix Reasoning scores were associated with thickening of the left middle frontal sulcus, superior frontal gyrus, and collateral sulcus, and right precentral sulcus, rectus gyrus, and lingual gyrus and at a trend level, another region of the right rectus gyrus and right orbital gyrus and paracentral sulcus. 


\section{Articles | Clairman et al.}

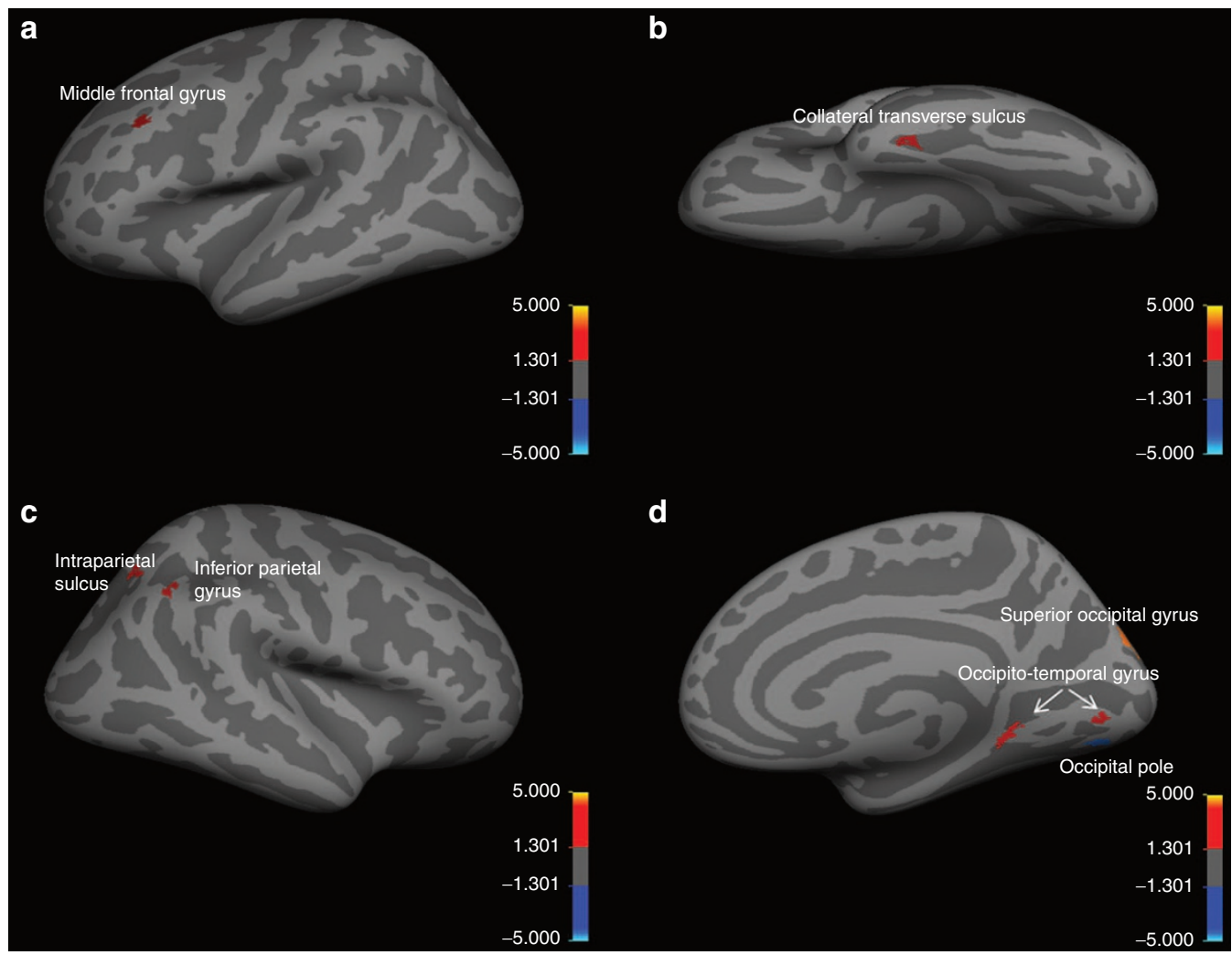

Figure 6. Comparison of cortical thickness between athyrotic and ectopic etiology subgroups. Red tones denote regions where athyrotic individuals are thicker relative to ectopic and blue shades where they are thinner. Left hemisphere lateral (a) and inferior (b) views; right hemisphere lateral (c) and medial (d) views. A $P$ value of $\leq 0.05$ was used for statistical significance.

Table 6. Regions showing significant correlations between neuropsychological test results and cortical thickness

\begin{tabular}{|c|c|c|c|c|c|c|}
\hline Hemisphere & $\begin{array}{l}\text { Lobe/ } \\
\text { region }\end{array}$ & Region & $\begin{array}{c}\text { Talairach } \\
\text { coordinates }(x, y, z)\end{array}$ & \# vertices & Size $\left(\mathrm{mm}^{2}\right)$ & $P$ value \\
\hline \multicolumn{7}{|c|}{ WASI full-2 IQ score } \\
\hline \multirow[t]{3}{*}{ Left } & Frontal & Anterior cingulate gyrus & $-6,33,8$ & 143 & 77.3 & 0.0076 \\
\hline & Frontal & Rectus gyrus & $-7,31,-21$ & 175 & 94.5 & 0.0016 \\
\hline & Frontal & Superior frontal gyrus & $-8,39,29$ & 153 & 107.5 & 0.0004 \\
\hline Right & Frontal & Orbital gyrus & $37,46,-6$ & 128 & 89.2 & 0.0044 \\
\hline \multicolumn{7}{|c|}{ WASI vocabulary scaled score } \\
\hline \multirow[t]{3}{*}{ Left } & Frontal & Middle frontal gyrus & $-34,16,48$ & 170 & 109.7 & 0.0002 \\
\hline & Frontal & Superior frontal gyrus & $-7,34,48$ & 171 & 113.3 & 0.0002 \\
\hline & Temporal & Collateral transverse sulcus & $-42,-19,-23$ & 465 & 250.8 & 0.0002 \\
\hline Left & Occipital & Collateral sulcus & $-23,-79,0$ & 125 & 104.2 & 0.0004 \\
\hline \multirow[t]{6}{*}{ Right } & Central & Paracentral sulcus & $14,-36,62$ & 189 & 70.3 & 0.0136 \\
\hline & Frontal & Orbital gyrus & $38,45,-6$ & 107 & 80.8 & 0.0044 \\
\hline & Frontal & Precentral sulcus & $27,-8,58$ & 207 & 99.8 & 0.0008 \\
\hline & Frontal & Rectus gyrus & $5,26,-21$ & 169 & 76.3 & 0.0060 \\
\hline & Frontal & Rectus gyrus & $6,13,-13$ & 282 & 133.8 & 0.0004 \\
\hline & Occipital & Lingual gyrus & $7,-75,3$ & 106 & 106.5 & 0.0008 \\
\hline
\end{tabular}




\section{DISCUSSION}

Our study sought to determine whether children with $\mathrm{CH}$ exhibit atypical cortical morphology, specifically abnormal CT, and if this reflects early hypothyroidism severity and later cognitive outcome. Compared with normothyroid controls, $\mathrm{CH}$ showed cortical thinning and cortical thickening in multiple regions of both hemispheres. Thinning was confined mainly to frontal, parietal, and temporal lobes, whereas thickening occurred throughout the cortex and primarily within sulci. Some, but not all, regions differentiating groups showed correlations with indices of initial disease severity, as measured by $\mathrm{T} 4$ and TSH levels at diagnosis, as well as $\mathrm{CH}$ etiology. Furthermore, cortical thickening of frontal, temporal, and occipital regions in $\mathrm{CH}$ was associated with poorer cognitive outcome.

Cortical thinning was observed in 11 regions of the frontal, parietal, and temporal lobes, primarily gyri or poles. These included the superior and middle frontal gyri and frontal pole, inferior temporal gyrus and temporal poles, and superior parietal gyrus and precuneus. Most effects were bilateral although some were confined to only the left (e.g., superior parietal gyrus) or right hemispheres (e.g., inferior temporal gyrus, precuneus). Cortical thinning related to initial hypothyroidism severity was seen in frontal and temporal regions, usually bilaterally. A comparison of athyrotic and ectopic etiological subgroups showed those with athyrosis exhibited greater thinning of the right occipital pole. As this is an early developing region (22), this effect may reflect greater availability of TH during gestation in the ectopic subgroup whose ectopic gland functioned partially vs. the athyrotic group whose gland was absent. Current observations on cortical thinning in general may be explained by findings from Mohan et al. (12) showing rodents with early TH deprivation had abnormal corticogenesis which reflected fewer neurons at the cortical plate.

Children with $\mathrm{CH}$ also showed cortical thickening in central (e.g., left central sulcus) and posterior (e.g., calcarine sulcus, middle and superior occipital sulci, occipital pole) regions. In contrast to thinning that was affected by perinatal hypothyroidism in only a handful of structures, thickening was observed more extensively and in both gyri and sulci. Our findings revealed thickening in the cingulate sulci and precentral, paracentral, and postcentral gyri of either or both hemispheres. When we examined $\mathrm{CH}$ etiology effects, children with athyrosis showed diverse thickening across multiple brain regions vs. children with ectopic glands. These findings may be attributed to the abnormal cortical migration patterns seen in rodents rendered hypothyroid gestationally wherein radial migration effects were decreased and tangential migration effects were increased (7). As some neurons ended up elsewhere than destined, certain cortical regions had a reduced number of neurons and others nearby, more than the requisite number of neurons. This concurs with our finding that $\mathrm{CH}$ had thinning in the superior frontal gyrus and thickening in the middle frontal sulcus. Current findings are also consistent findings of local migration abnormalities in sensorimotor, auditory, and visuospatial cortices of TH-deprived rodents $(11,23)$.
Alternatively, increased $\mathrm{CT}$ in $\mathrm{CH}$ may have arisen from disturbances in eliminating transitory ipsi- and contra-lateral connections within the cortex because of the lengthened period of maturation from their hypothyroidism (24). In other words, cortical thickening may represent a more juvenile pattern of brain development than seen in TDC. Additionally, some neurons failing to reach the cortical plate may have remained in lower layers or even the white matter, thus giving the appearance of an abnormally long-and hence thicker-cortex (11). Also, these migrational failures could lead to heterotopias, as observed in autism (25), which coincidentally is also associated with gestational TH insufficiency (26). Another possibility is that increased cortical thickening reflects reduced pruning due to attenuated apoptosis or programmed cell death, as seen in TH-deficient rodents (12). Moreover, events beyond the time of peak hypothyroidism (i.e., right after birth) may also be contributing to findings. For example, the duration of hypothyroidism until euthyroidism is achieved may be manifested later in infancy when some brain networks are becoming refined (27). As with thinning, comparison of athyrotic and ectopic subgroups showed differences primarily in posterior regions. This may reflect earlier neurodevelopmental effects in gestation when some TH was available for the ectopic but not athyrotic subgroup (6).

Our findings of different association patterns between regions showing thinning or thickening and early hypothyroidism severity (low T4dx or high TSHdx) may reflect the particular developmental stage of a specific structure when $\mathrm{TH}$ was measured, given nonuniform brain development and need for TH within the brain. Since brain development usually proceeds in a posterior-to-anterior fashion (28) and may differ between hemispheres (29), our observation that groups differed most and correlations were strongest in frontal regions and the right hemisphere may signify these brain regions were undergoing maximal development at the time of greatest $\mathrm{TH}$ deficiency in our sample.

Several explanations may also account for the discrepancies in correlations between CT and T4dx vs. TSHdx. First, both hormones operate at different levels given that TSH reflects availability of hormone within the brain, whereas T4 reflects hormone in serum and not necessarily at the neuronal level given the number of steps needed to convert T4 to T3 (30) and transport $\mathrm{T} 4 / \mathrm{T} 3$ across membranes within the brain (31). These steps, which can differ among individuals, were not currently measured.

Incomplete overlap between the group and correlational analysis results may reflect that for group analyses, effects were somewhat muted by including less affected $\mathrm{CH}$ children, who are also contributing the large variance seen in the correlational analyses. Since more regions showed thinning in the between-group comparisons than correlations with indices of severity at time of diagnosis (viz., at $~ 2$ wk of life), it is possible that other factors such as when the hypothyroidism first appeared or how long it lasted may be accounting for the group differences. Because the frontal lobes need TH last during development (32), observed frontal thinning in $\mathrm{CH}$ 


\section{Articles | Clairman et al.}

may reflect a deficiency of TH long after time of diagnosis but before euthyroidism was ultimately achieved. Likewise, more posterior effects when etiologic $\mathrm{CH}$ subgroups were contrasted may reflect the gestational loss of TH experienced by the athyrotic subgroup primarily.

It is also possible that the observed $\mathrm{CH}$ thinning and thickening may reflect events at time of brain scanning, not infancy, including a slower continuous rate of neurodevelopmental maturation. Since adolescence is when critical CT changes occur that reflect thickening followed by thinning, the $\mathrm{CH}$ group's effects may reflect their experiencing a potentially slower rate of maturation. This could be set in motion by their early TH loss or by lengthy periods of low TH between scheduled endocrine clinic visits and receiving dose increments. Shaw et al. (22) reported a relationship between CT and age whereby some cortical regions showed a continuous linear increase in CT and others showed a curvilinear relationship reflecting thickening followed by thinning. Furthermore, age when peak thickening occurred and thinning began was seen to differ among brain regions (22) with some as early as age 6 (right occipital pole) and others as late as age 15 (bilateral superior temporal gyrus) or even age 18 (bilateral insula). Our diverse effects may therefore reflect what proportion of each group is transitioning from a mode of predominantly thickening to one of predominantly thinning. Interestingly, when we conducted a post-hoc analysis using the ages of peak CT cited by Shaw et al. (22), those regions where $\mathrm{CH}$ showed thickening relative to TDC were ones with earlier peaks than those showing thinning relative to TDC. Consequently for earlier developing regions, $\mathrm{CH}$ were lagging behind TDC and still undergoing thickening, and for later developing regions, they had not yet achieved the same degree of thinning as TDC, and so appeared thicker.

Several research groups have reported sexual dimorphisms in CT for certain regions, although effects are inconsistent and differ by brain region (33). Sowell et al. (34), for example, reported females had thicker cortices than males in right inferior parietal and posterior temporal regions and, to a lesser degree, left posterior temporal and left ventral frontal regions; in contrast, males demonstrated greater cortical thickening than females in right anterior temporal and orbitofrontal regions. Because our sample was matched for sex and had roughly similar distributions of males and females, it is unlikely that sex differences between groups can account for our findings. Nevertheless, as recent evidence on rodents suggests the possibility of a sex difference in molecular response to TH deficiency within the brains of male vs. female mice (35), analyses of sex differences are certainly warranted for future CT studies on this population.

Recently, a number of studies using FreeSurfer (Laboratory for Computational Neuroimaging, Martinos Center for Biomedical Imaging) or a similar tool have been conducted on other populations of the same age range as ours. For example, cortical thinning was seen in 22q11.2 deletion syndrome (36) and autism (14) and cortical thickening in fetal alcohol spectrum disorder (17) and ADHD (37) while both thinning and thickening were seen in autism (38), prematurity (15), and ADHD (16). Although it is not readily clear what accounts for this variation, even within the same disorder, this may reflect the particular analytic tool, statistical approach, or sample characteristics (e.g., age range, sex composition). Nevertheless among these other clinical populations, no clear pattern emerges as to when effects of insult (e.g., prenatal, postnatal) or onset of disorder (infancy, childhood) affect cortical morphology.

Since the advent of newborn screening, extensive research has revealed the early $\mathrm{TH}$ deficiency in $\mathrm{CH}$ contributes to a variety of persisting cognitive deficits in language, sensorimotor, visuospatial, memory, and attention areas. We currently observed CT abnormalities in many brain regions known to be associated with these abilities. For example, weaknesses in sensorimotor skills, which rely on an intact primary somatosensory cortex, concur with our findings of cortical thickening in the left central sulcus. Similarly, our observation of thinning within the left superior parietal gyrus and right precuneus in $\mathrm{CH}$ is consistent with their visuospatial difficulties $(39,40)$. Likewise, $\mathrm{CH}$ deficits on lower-order and higher-order visual perception tasks (39) may reflect cortical abnormalities in temporal and occipital regions, as indicated by thinning in their right inferior temporal region and thickening in their left calcarine sulcus and right middle and superior occipital sulcus, all of which are known to support visuoperceptual abilities. Also, our findings of bilateral cortical thinning in middle and superior frontal regions may explain $\mathrm{CH}$ weaknesses on working memory tasks (41).

\section{Limitations}

Our sample included a few $\mathrm{CH}$ participants who lacked $\mathrm{TH}$ data and several late-treated cases who were diagnosed out of country, missed by the screening program, or assigned to wait until T4 levels dropped below normal. Our TDC group may have been biased since some particularly interested in participating in research had higher-than-normal functioning while others had an undisclosed problem (e.g., past concussion) for which mothers were seeking a free scan. Our broad age range may have occurred when marked CT changes reflecting thickening followed by thinning took place (22); thus some CT variability may be reflecting exactly when the child was tested, not the condition. Also, extraneous information (e.g., enrichment) can influence CT results, particularly given our findings of larger hippocampi among $\mathrm{CH}$ children who received music training (42); such influences were not recorded presently. Although we compared only athyrotic and ectopic subgroups given the small number of cases with dyshormonogensis, the latter group who tends to be treated relatively late, may have been driving some of the group results. Additionally, correlations were computed only with TSHdx and T4dx and not other indices of earlier or later TH loss (e.g., bone age, time to euthyroidism), or current TH levels. For TDC, TH data were obtained for only 
a subset (i.e., participants in one of the three studies), none of whom were hypothyroid.

Regarding our methods, we were limited to just a handful of tests common across the various studies from which our large sample was derived. Consequently, we could not examine for critical structure-function correlations. In addition, FreeSurfer (Laboratory for Computational Neuroimaging, Martinos Center for Biomedical Imaging) is limited by (i) being a strictly automated technique that can over- or underestimate some regions, (ii) using an adult brain atlas for regional parcellation when our subjects were pre- or early adolescent, and (iii) labelling clusters of peak difference or correlation separately despite some falling in very close proximity. Also, we did not examine for other aspects of cortical morphology that may also be sensitive to lack of $\mathrm{TH}$ such as surface area or gyrification. Results from these further analyses will be forthcoming in a future paper.

\section{Conclusion}

This study provides novel evidence showing human cortical development is definitely compromised by $\mathrm{CH}$. Relative to TDC, children with $\mathrm{CH}$ were seen to exhibit both cortical thinning and cortical thickening in diverse brain regions subserving some of the deficient abilities of this population. Additionally, some regions showing abnormal cortical morphology were those directly correlated with severity of hypothyroidism at diagnosis while increased CT in some brain regions was associated with poorer neuropsychological outcome. Overall, the current research fills a critical knowledge gap by showing that the brief but circumscribed period of TH deficiency that children with $\mathrm{CH}$ still undergo has an impact on their cortical development. Moreover, as our findings are consistent with those on animals showing abnormalities in asymmetrical division and neuronal migration, this offers an explanatory mechanism for the suboptimal outcome seen in $\mathrm{CH}$. Directions for future research include examining $\mathrm{CT}$ differences in all $\mathrm{CH}$ etiologies, sex differences, exact age and stage of pubertal development at time of scanning, as well as other cortical morphology metrics.

\section{METHODS}

\section{Participants}

The $\mathrm{CH}$ group consisted of 42 participants (20 males and 22 females) from three prior studies, each involving a neuroimaging component. Participants ranged in age from 9.3 to $16.8 \mathrm{y}$ and were derived from a longitudinal cohort followed since birth or were recruited recently from the Endocrine Clinic at The Hospital for Sick Children (SickKids). Although the majority of cases were diagnosed by the Ontario newborn screening program and treated very early in life, a few children were diagnosed out of country and only first treated at about 1 mo of age while one Ontario-born boy was missed by the newborn screening program and did not start treatment until $62 \mathrm{~d}$. Several others with very mild TSH elevations were followed only until their T4 levels fell below normal, and then were treated. For most children, medical charts contained the requisite information on CH etiology, T4dx and TSHdx, age at treatment onset, and initial treatment dose. Thirteen children had athyrosis, 20 had ectopic glands, and 7 had dyshormonogenesis; etiology was unknown in two cases.

The control sample, which originally consisted of 52 participants from the same three studies as the $\mathrm{CH}$ group, was derived either from our longitudinal cohort born 1996 to 2001 or recently recruited by local advertising. Eligibility was based on a prescreening telephone interview with parents to establish if their child (i) was free of major health problems (including an endocrine abnormality), (ii) did not have a learning disability, attention disorder, head injury, or a contraindication to scanning (e.g., braces or dental implants), and (iii) was willing to undergo scanning. Only children whose scans showed neither excessive motion nor an incidental finding in the neuroradiologist's report were included. From these, 42 were selected to match those in the $\mathrm{CH}$ group for sex and age range.

Procedures for the various studies from that the scans were retrieved were approved by the SickKids Research Ethics Board. Informed consent was obtained from parents of all children who participated in the studies.

\section{Tests and Measures}

All children received a variety of neuropsychological tests, that depended on the particular study from which they were ascertained. Across studies, the only tests in common were the WASI, based on Vocabulary and Matrix Reasoning subtests, and the REY-O. WASI Vocabulary assesses expressive word knowledge by having the child define words and concepts presented orally or pictorially while WASI Matrix Reasoning assesses nonverbal reasoning by requiring the child to identify from four choices, one that completes a figure or pattern. Scores are given as T-scores $($ mean $=50 ; S D=10)$ while their combination is converted to a standard score $($ mean $=100 ; S D=15)$ that serves as an approximation of IQ. REY-O requires reproducing a complex abstract figure (copy condition) in sight and after a 20-min delay (delayed condition); results are given as Z-scores (mean $=0$; $\mathrm{SD}=1$ ) based on normative data provided. Handedness was recorded in all children except one $\mathrm{CH}$ and two TDC.

Tests were administered by psychometrists and advanced graduate students uniformly trained in the procedures and masked to participant group.

\section{MRI Acquisition and Analysis}

All imaging took place in the 1.5 Tesla GE Signa Excite research scanner at SickKids. Total scan time was $1 \mathrm{~h}$ per child to allow for acquisition of the various sequences for each study, as well as the neuroradiological scans. For current purposes, we used an anatomical Inversion Recovery Prepared T1-weighted FSPGR sequence with the following acquisition parameters: repetition time $=10.09 \mathrm{~ms}$, echo time $=4.2 \mathrm{~ms}$, inversion time $=400 \mathrm{~ms}$, flip angle $=20^{\circ}$, and acquisition matrix $=256 \times 192 \mathrm{~mm}$. Voxel sizes were $0.9375 \times 0.9375 \mathrm{~mm}$ and slice thickness was $1.5 \mathrm{~mm}$.

Automated cortical reconstruction of the T1-weighted images was performed on a Linux Fedora operating system using FreeSurfer version 4.4 (Laboratory for Computational Neuroimaging, Martinos Center for Biomedical Imaging), which is widely documented and freely available online. Processing involved several stages described at length (13). Briefly, this included transformation of each subject's native brain into Talairach space, intensity normalization, removal of nonbrain tissue, and segmentation of the gray matter/white matter (GM/WM) tissue. Every cortex was also manually inspected for quality control. Cortical thickness was calculated as the shortest distance between the pial surface and GM/WM boundary at each vertex of the entire cortical mantle of each hemisphere. Also provided by FreeSurfer (Laboratory for Computational Neuroimaging, Martinos Center for Biomedical Imaging) was a measure of intracranial, gray matter, white matter, and cerebrospinal fluid volume for each subject.

\section{Data Management and Statistical Plan}

We adopted an intent-to-treat model approach, whereby every case entered was included. Since only 27 children in the $\mathrm{CH}$ group had complete biomedical data, we needed to replace any missing biomedical information. We used a systematic multi-facetted approach that approximated each child's biology from data in his or her medical chart and took into account the initial degree of severity, which is known to vary by etiology (43). For five cases with a TSHdx but a free T4 instead of total T4dx (since the first set of blood tests were obtained at an outside lab not SickKids), we estimated their T4dx by computing a total:free T4 ratio from child's next SickKids visit and applied this back to the free 
T4dx value. For six cases with a TSHdx value but neither a total T4 or free T4 value at diagnosis (three athyrosis, one dyshormonogenesis, two ectopic), we performed regressions between TSHdx and total T4dx for all members of each etiological group and then used resulting regression parameters to derive total T4 values from child's TSHdx value. For cases lacking both T4 and TSH data $(n=4)$, we used the child's etiological group mean values, if known $(n=3)$, or the total sample's mean values if etiology was not known $(n=1)$.

Chi-square and independent samples $t$-tests served to compare groups for demographic and neuropsychological test results using SPSSv21. Although we planned to adjust for age or sex differences using covariates, this was not done as these parameters did not significantly differentiate groups. MRI data were statistically analyzed within FreeSurfer (Laboratory for Computational Neuroimaging, Martinos Center for Biomedical Imaging) using a general linear model at the vertex-wise level on left and right hemispheres separately. Two-tailed $t$-tests served to compare groups and etiologic subgroups while correlations were performed between regional CT values and biomedical (T4dx, TSHdx) and behavioral indices (WASI IQ, Vocabulary, Matrix Reasoning, and REY-O Copy and Delayed). A cluster-wise procedure using the Monte Carlo Null-Z simulation (44) with 5,000 permutations was used to correct maps for multiple comparisons; a threshold of $P<0.05$ was allowed. For all analyses, the Destrieux Atlas was used to identify locations of significant clusters (45).

\section{ACKNOWLEDGMENTS}

Authors would like to thank Anishka Greene, Dragana Ostojic, Erin Sheard, Michelle Vitti, Sarah Wheeler, and Karen Willoughby for their assistance in data collection for this study; Garry Detzler, Tammy Rayner, and Ruth Weiss for obtaining MR scans; and Susan Blaser for reading the neuroradiological scans. Our extreme gratitude goes to the children and families whose dedication to this research and willingness to participate would not have made these findings possible. Finally, we thank two anonymous reviewers for their insightful comments on an earlier draft of this paper.

\section{STATEMENT OF FINANCIAL SUPPORT}

This study was funded by a Canadian Institutes of Health Research operating grant (MOP 49488).

Disclosure: Authors have no financial ties or conflicts of interest to disclose.

\section{REFERENCES}

1. Harris KB, Pass KA. Increase in congenital hypothyroidism in New York State and in the United States. Mol Genet Metab 2007;91:268-77.

2. Lania A, Persani L, Beck-Peccoz P. Central hypothyroidism. Pituitary 2008;11:181-6.

3. Rovet J. Congenital hypothyroidism: treatment and outcome. Curr Opin Endocrinol Diabetes Obes 2005; 12:42-52.

4. Rovet JF, Ehrlich R. Psychoeducational outcome in children with earlytreated congenital hypothyroidism. Pediatrics 2000;105(3 Pt 1):515-22.

5. Tinelli F, Costagli C, Bargagna S, Marcheschi M, Parrini B, Perelli V. Behavioural disorders in adolescents with early-treated congenital hypothyroidism. Funct Neurol 2003;18:161-4.

6. Rovet J, Daneman D. Congenital hypothyroidism: a review of current diagnostic and treatment practices in relation to neuropsychologic outcome. Paediatr Drugs 2003;5:141-9.

7. Cuevas E, Ausó E, Telefont M, Morreale de Escobar G, Sotelo C, Berbel P. Transient maternal hypothyroxinemia at onset of corticogenesis alters tangential migration of medial ganglionic eminence-derived neurons. Eur J Neurosci 2005;22:541-51.

8. Rami A, Patel AJ, Rabié A. Thyroid hormone and development of the rat hippocampus: morphological alterations in granule and pyramidal cells. Neuroscience 1986;19:1217-26.

9. Gil-Ibañez P, Morte B, Bernal J. Role of thyroid hormone receptor subtypes $\alpha$ and $\beta$ on gene expression in the cerebral cortex and striatum of postnatal mice. Endocrinology 2013;154:1940-7.

10. Berbel PJ, Escobar del Rey F, Morreale de Escobar G, Ruiz-Marcos A. Effect of hypothyroidism on the size of spines of pyramidal neurons of the cerebral cortex. Brain Res 1985;337:217-23.
11. Berbel P, Navarro D, Ausó E, et al. Role of late maternal thyroid hormones in cerebral cortex development: an experimental model for human prematurity. Cereb Cortex 2010;20:1462-75.

12. Mohan V, Sinha RA, Pathak A, et al. Maternal thyroid hormone deficiency affects the fetal neocorticogenesis by reducing the proliferating pool, rate of neurogenesis and indirect neurogenesis. Exp Neurol 2012;237:477-88.

13. Fischl B, Dale AM. Measuring the thickness of the human cerebral cortex from magnetic resonance images. Proc Natl Acad Sci USA 2000;97: 11050-5.

14. Wallace GL, Dankner N, Kenworthy L, Giedd JN, Martin A. Age-related temporal and parietal cortical thinning in autism spectrum disorders. Brain 2010;133(Pt 12):3745-54.

15. Martinussen M, Fischl B, Larsson HB, et al. Cerebral cortex thickness in 15 -year-old adolescents with low birth weight measured by an automated MRI-based method. Brain 2005;128(Pt 11):2588-96.

16. Almeida Montes LG, Prado Alcántara H, Martínez García RB, De La Torre LB, Avila Acosta D, Duarte MG. Brain cortical thickness in ADHD: age, sex, and clinical correlations. J Atten Disord 2013;17:641-54.

17. Yang Y, Roussotte F, Kan E, et al. Abnormal cortical thickness alterations in fetal alcohol spectrum disorders and their relationships with facial dysmorphology. Cereb Cortex 2012;22:1170-9.

18. Wheeler SM, Willoughby KA, McAndrews MP, Rovet JF. Hippocampal size and memory functioning in children and adolescents with congenital hypothyroidism. J Clin Endocrinol Metab 2011;96:E1427-34.

19. Wheeler SM, McAndrews MP, Sheard ED, Rovet J. Visuospatial associative memory and hippocampal functioning in congenital hypothyroidism. J Int Neuropsychol Soc 2012;18:49-56.

20. Wechsler D. Wechsler Abbreviated Scale of Intelligence. New York: Psychological Corp, 1999.

21. Strauss E, Serman EMS, Spreen O. A Compendium of Neuropsychological Tests. 3rd edn. New York: Oxford University Press, 2006.

22. Shaw P, Kabani NJ, Lerch JP, et al. Neurodevelopmental trajectories of the human cerebral cortex. J Neurosci 2008;28:3586-94.

23. Berbel P, Guadaño-Ferraz A, Angulo A, Ramón Cerezo J. Role of thyroid hormones in the maturation of interhemispheric connections in rats. Behav Brain Res 1994;64:9-14.

24. Berbel P, Navarro D, Román GC. An evo-devo approach to thyroid hormones in cerebral and cerebellar cortical development: etiological implications for autism. Front Endocrinol (Lausanne) 2014;5:146.

25. Stoner R, Chow ML, Boyle MP, et al. Patches of disorganization in the neocortex of children with autism. N Engl J Med 2014;370:1209-19.

26. Román GC, Ghassabian A, Bongers-Schokking JJ, et al. Association of gestational maternal hypothyroxinemia and increased autism risk. Ann Neurol 2013;74:733-42.

27. Huttenlocher PR, Dabholkar AS. Regional differences in synaptogenesis in human cerebral cortex. J Comp Neurol 1997;387:167-78.

28. Giedd JN, Blumenthal J, Jeffries NO, et al. Brain development during childhood and adolescence: a longitudinal MRI study. Nat Neurosci 1999;2:861-3.

29. Zhou D, Lebel C, Evans A, Beaulieu C. Cortical thickness asymmetry from childhood to older adulthood. Neuroimage 2013;83:66-74.

30. Hernandez A, Morte B, Belinchón MM, Ceballos A, Bernal J. Critical role of types 2 and 3 deiodinases in the negative regulation of gene expression by $\mathrm{T}_{3}$ in the mouse cerebral cortex. Endocrinology 2012;153: 2919-28.

31. Müller J, Heuer H. Expression pattern of thyroid hormone transporters in the postnatal mouse brain. Front Endocrinol (Lausanne) 2014;5:92.

32. Gould E, Butcher LL. Developing cholinergic basal forebrain neurons are sensitive to thyroid hormone. J Neurosci 1989;9:3347-58.

33. Lenroot RK, Giedd JN. Sex differences in the adolescent brain. Brain Cogn 2010;72:46-55.

34. Sowell ER, Peterson BS, Kan E, et al. Sex differences in cortical thickness mapped in 176 healthy individuals between 7 and 87 years of age. Cereb Cortex 2007;17:1550-60.

35. Dong H, Wade M, Williams A, Lee A, Douglas GR, Yauk C. Molecular insight into the effects of hypothyroidism on the developing cerebellum. Biochem Biophys Res Commun 2005;330:1182-93. 
36. Bearden CE, van Erp TG, Dutton RA, et al. Mapping cortical thickness in children with 22q11.2 deletions. Cereb Cortex 2007;17:1889-98.

37. Duerden EG, Tannock R, Dockstader C. Altered cortical morphology in sensorimotor processing regions in adolescents and adults with attentiondeficit/hyperactivity disorder. Brain Res 2012;1445:82-91.

38. Mak-Fan KM, Taylor MJ, Roberts W, Lerch JP. Measures of cortical grey matter structure and development in children with autism spectrum disorder. J Autism Dev Disord 2012;42:419-27.

39. Simic N, Khan S, Rovet J. Visuospatial, visuoperceptual, and visuoconstructive abilities in congenital hypothyroidism. J Int Neuropsychol Soc 2013;19:1119-27.

40. Blasi V, Longaretti R, Giovanettoni C, et al. Decreased parietal cortex activity during mental rotation in children with congenital hypothyroidism. Neuroendocrinology 2009;89:56-65.
41. Song SI, Daneman D, Rovet J. The influence of etiology and treatment factors on intellectual outcome in congenital hypothyroidism. J Dev Behav Pediatr 2001;22:376-84.

42. Zendel BR, Willoughby KA, Rovet JF. Neuroplastic effects of music lessons on hippocampal volume in children with congenital hypothyroidism. Neuroreport 2013;24:947-50.

43. Hanukoglu A, Perlman K, Shamis I, Brnjac L, Rovet J, Daneman D, Relationship of etiology to treatment in congenital hypothyroidism. J Clin Endocrinol Metab 2001;86:186-91.

44. Hagler DJ Jr, Saygin AP, Sereno MI. Smoothing and cluster thresholding for cortical surface-based group analysis of fMRI data. Neuroimage 2006;33:1093-103.

45. Fischl B, van der Kouwe A, Destrieux C, et al. Automatically parcellating the human cerebral cortex. Cereb Cortex 2004;14:11-22. 